\title{
O SETOR ESPACIAL BRASILEIRO À LUZ DAS RECENTES MUDANÇAS DOS ARRANJOS ORGANIZACIONAIS
}

\author{
Marcos José Barbieri Ferreira e Celso Neris Jr.*
}

\begin{abstract}
Área 1: Dinâmicas industriais setoriais e dos sistemas de produção
Área 5: Competências e capacitações das empresas
\end{abstract}

\begin{abstract}
Resumo: Este artigo busca avaliar a participação das empresas brasileiras nos programas espaciais que vêm sendo executados no Brasil, particularmente no que se refere à capacitação tecnológica, produtiva e organizacional dessas empresas. Utilizando como fonte primária os dados do Mapeamento da Indústria Espacial Brasileira, realizado no ano de 2013 para o Centro de Gestão e Estudos Estratégicos (CGEE), são descritas e analisadas as capacitações e limitações das empresas brasileiras que vêm participando dos diferentes programas espaciais realizados no país. É possível constatar que estas empresas possuem competências tecnológicas restritas à segmentos de mercado bastante específicos, implicando na especialização e pulverização da estrutura produtiva do setor. Além disso, restrições produtivas e financeiras relacionadas às descontinuidades dos projetos espaciais são também percebidas. Neste sentido, a criação da Visiona, em conjunto com INPE e DCTA, como contratante e integradora de um novo programa de satélites constitui uma mudança na estrutura organizacional que procuraremos também avaliar.
\end{abstract}

Palavras-chaves: indústria espacial; capacitações; arranjo organizacional

\begin{abstract}
This article seeks to evaluate the participation of brazilian firms in the space programs that are being implemented in Brazil, particularly in relation to technological, productive and organizational capacity of these companies. Using as a primary source the data of Mapeamento da Indústria Espacial Brasileira, held in 2013 for the Center for Strategic Studies and Management Science, Technology and Innovation (CGEE), we describe the capabilities and limitations of brazilian firms that are participating of space programs conducted in Brazil. It can be seen that these companies have technological skills restricted to very specific market segments, resulting in specialization and in the spraying of the productive structure of the sector. Moreover, productive and financial constraints related to discontinuities of space projects are also perceived. In this sense, the creation of Visiona, together with INPE and DCTA, as contractor and integrator of a new satellite program is a change in the organizational structure that we also seek to evaluate.
\end{abstract}

Palavras-chaves: space industry; capacitations; organisational arrangements

JEL: L60, L10, D24

\footnotetext{
* Respectivamente, Professor da Faculdade de Ciências Aplicadas da Unicamp (FCA/Unicamp) e doutorando em Teoria Econômica pelo Instituto de Economia da Unicamp (IE/Unicamp) e pesquisador do Grupo de Estudos em Economia Industrial (GEEIN/UNESP). Ambos são pesquisadores do Laboratório de Estudos das Indústrias Aeroespaciais e de Defesa (LabA\&D) Emails: marcosbarbieriferreira@gmail.com e cnerisjr@gmail.com.
} 


\section{Introdução}

O segmento espacial de um país agrega uma vasta rede de instituições e atividades econômicas que envolvem produtos e serviços com aplicações em diferentes campos, que vão desde a transmissão de dados e previsões meteorológicas até o controle de tráfego aéreo e à defesa nacional (Matos, 2016). As principais motivações dos Estados para investirem na atividade espacial são a geração de vantagens competitivas, o desenvolvimento científico e tecnológico, o crescimento econômico, o monitoramento do meio ambiente, o prestígio global e a segurança nacional, por meio do poder estratégico oferecido pelo espaço (Futron, 2012).

De acordo com a literatura, os programas espaciais envolvem atividades em torno de três eixos: i. veículos lançadores; ii. satélites, que podem ser divididos em serviços, plataformas e cargas úteis; e iii. solo, compreendendo as estações de rastreio e controle dos satélites e os centros de missão para o gerenciamento das cargas úteis dos satélites (Gattaz et al, 2012). As principais características dos programas espaciais são ressaltadas por Vaz (2011): a) os recursos destes programas são de natureza governamental; b) o mercado é altamente competitivo e os riscos são elevados; c) os bens são desenvolvidos por encomendas e possuem alto valor agregado; d) envolvem tecnologias classificadas como “duais” (militares e civis); e) os custos de treinamento e especialização da mão de obra são muito elevados, e f) o mercado é limitado em quantidade de encomendas. Todos estes elementos justificam uma política governamental de aquisição que privilegie as empresas nacionais. Sendo assim, o papel do Estado é fundamental, uma vez que ele pode atuar como demandante e financiador das atividades espaciais, além de promover a cooperação entre instituições públicas e privadas. Desta maneira, o Estado fomenta o desenvolvimento de atividades científica e tecnológicas em setores de alto valor agregado.

Neste contexto, Matos (2016) cita o recente avanço da globalização das atividades espaciais. Segundo a autora, há duas décadas apenas os Estados Unidos, a Rússia, a Europa e a China tinham regularmente lançado e implementado satélites e outras missões no espaço. Nos dias atuais, várias nações emergentes realizam atividades espaciais e vêm buscando efetivar acordos de cooperação com o objetivo de desenvolver ainda mais as respectivas indústrias espaciais.

De maneira geral, as tecnologias da indústria espacial estão relacionadas à duas grandes atividades: a fabricação de foguetes e de satélites e os serviços de lançamento (Dewes, 2012). Carvalho (2011) argumenta que a construção de satélites, de foguetes e de infraestrutura terrestre apresenta complexidade e riscos tecnológicos, altos custos e ciclos de desenvolvimento longos, devido à elevada complexidade dos produtos espaciais. Em decorrência disso, a classificação da indústria espacial assume uma diversidade de formas, dependendo das suas aplicações, o que implica na impossibilidade de se estabelecer uma classificação com base na Classificação Nacional de Atividades Econômicas (CNAE). A Space Industry Association (SIA), por exemplo, associa a indústria espacial à indústria de satélites (Matos, 2016). Segundo a SIA, a indústria de satélites representou cerca de 62\% das receitas da atividade espacial no mundo em 2012 e o restante das atividades espaciais estava vinculado, principalmente, aos programas governamentais (Matos, 2016). Nesse texto, o setor pode ser definido, em termos de oferta de produtos, por empresa que produzem satélites, veículos lançadores e seus respectivos sistemas e subsistemas.

Com isto, busca-se avaliar a participação das empresas brasileiras nos recentes programas espaciais nacionais, particularmente no que se refere a capacitação tecnológica, produtiva e organizacional dessas empresas, além de avaliar as recentes mudanças nos arranjos organizacionais, particularmente, o estabelecimento da Visiona como a primeira empresa integradora e contratante do setor espacial brasileiro. O trabalho utilizará informações de fontes secundárias e primárias. Além de complementar a descrição e análise das empresas, as fontes secundárias complementarão a revisão bibliográfica previamente realizada. Contudo o destaque está nas fontes primárias que utilizarão a base de dados do Mapeamento da Indústria Espacial Brasileira, um levantamento recentemente realizado para o Centro de Gestão e Estudos Estratégicos (CGEE). Este mapeamento foi empreendido através de visitas técnicas e entrevistas junto às principais empresas da indústria 
espacial brasileira. Uma ressalva a ser feita é que algumas informações sobre o setor apresentam restrições. Primeiro, por envolverem os movimentos estratégicos de longo prazo das principais empresas do setor e; segundo, por se tratar de um setor que ocupa um lugar de destaque na estrutura de defesa, sendo assim um setor estratégico-militar.

O texto está dividido em duas seções, além desta introdução. Na primeira seção é descrita a dinâmica tecnológica do segmento espacial, com base na literatura neoschumpeteriana, e o seu impacto sobre os arranjos institucionais. A segunda seção trata do setor espacial brasileiro e está subdividida em duas partes. Na primeira, elaborada com base nas empresas selecionadas e utilizando-se da metodologia anteriormente citada, são analisadas as principais empresas fornecedoras do setor espacial brasileiro. Na segunda parte, há uma caracterização das instituições de pesquisa responsáveis pela integração, montagem e testes das plataformas espaciais discutindo, em sequência, as implicações das recentes mudanças organizacionais no setor, principalmente com a criação da empresa Visiona. Por fim, são apresentadas as breves considerações finais.

\section{Setor Espacial: A Dinâmica Tecnológica e os Arranjos Organizacionais}

A indústria espacial se caracteriza por produtos de elevado valor adicionado, empregos de alta qualificação e grande dinamismo tecnológico (Ferreira, 2010). As principais áreas de demandas do setor espacial - com destaque para comunicação, monitoramento e, mais recentemente, sistemas de geoposicionamento - buscam equipamentos cada vez mais avançados, precisos e com menores custos. No caso dos equipamentos espaciais de uso militar, a disputa estratégica pela primazia na incorporação de novas tecnologias impulsionam ainda mais as inovações do setor. Por fim, as demandas da área ciêntificas estão, por princípio, direcionadas para produtos inovadores. Nesse sentido, contata-se que o setor espacial precisa incorporar as mais sofisticadas tecnologias, posicionado-se permanentemente na fronteira tecnológica. Diante disso, a estrutura produtiva do setor espacial caracteriza-se por elevados investimentos em pesquisa e desenvolvimento, utilização intensiva de recursos humanos altamente qualificados e a integração de conhecimentos e atividades multidisciplinares.

As plataformas espaciais, particularmente satélites e veículos lançadores, são na realidade equipamentos complexos que integram de maneira organizada e lógica um conjunto de sistemas altamente sofisticados. Alguns desses sistemas são intrínsecos a própria plataforma - como as estruturas e os sistemas de propulsão, energia e atitude - , enquanto outros são específicos para a missão da plataforma, como sensores, câmeras e transponders. Outra importante característica destas plataformas é sua pequena escala produtiva, sendo equipamentos feitos basicamente sob encomenda ${ }^{1}$. Em razão disso, as plataformas espaciais apresentam alta densidade tecnológica, longo ciclo de desenvolvimento e manufatura, elevada complexidade na integração dos sistemas, resultando em custos e incertezas elevados.

Estas características fazem com que as plataformas espaciais também sejam classifcadas como sistemas intensivos em engenharia ou sistemas complexos (Miller et al, 1995). Segundo Chagas et al (2011, p. 64), os sistemas complexos caracterizam-se pela i) inexistência de um projeto dominante selecionado pelo mercado ex-post (Utterback, 1996; Ferreira, 2009); ii) a necessidade do domínio de uma grande quantidade de disciplinas tecnológicas, sendo estas integradas seguindo a lógica hierárquica dos sistemas complexos; iii) o estabelecimento necessário de redes interorganizacionais com base em um projeto e iv) o grande envolvimento de clientes ao longo de todo o processo de desenvolvimento. Neste sentido, a eficiência na gestão das relações interfirmas — arranjos organizacionais — é uma competência essencial para o desenvolvimento do setor.

Hobday (1998) faz uma oposição entre sistemas de produção em massa e os sistemas complexos. Os sistemas complexos, em relação aos produtos, são caracterizados por um sistema

\footnotetext{
${ }^{1}$ Isto explica a baixa automação da estrutura produtiva do setor espacial.
} 
hierárquico que desempenha várias funções, sendo comum a ocorrência de propriedades emergentes não planejadas. Quanto à produção, tem como base o projeto, ou pequenos lotes de produção, que envolvem mais de uma organização. No processo de inovação, há uma definição ex-ante pelas organizações, por meio de esforços cooperativos e tem papel relevante o usuário. Nas estratégias competitivas, há a gestão de alianças multi-firmas com base em projetos tendo como compromisso principal o desempenho competitivo dos seus produtos. Com relação a esse último item, destaca-se as capacitações de integração de sistemas entre as diferentes firmas.

Nesse ambiente, a integração de sistemas é a atividade fundamental. Hobday et al. (2005) destaca que a integração possui duas faces. A primeira diz respeito as atividades internas das empresas - como elas se desenvolvem e integram os insumos de que necessitam para produzir novos sistemas. A segunda, que se tornou a mais importante nos últimos anos, refere-se às atividades externas de empresas - como elas integram componentes, habilidades e conhecimentos de outras organizações ${ }^{2}$ para produzir sistemas cada vez mais complexos. Cabe ressaltar que quanto mais complexa, de alta tecnologia e de alto custo for a integração de sistemas, mais significativa ela será para a atividade da empresa e do conjunto do setor.

Sendo assim, os sistemas complexos são caracterizados por uma base tecnológica extremamente ampla, que reúne um conjunto de diferentes trajetórias tecnológicas que evoluem, com dinâmicas específicas, bem como a própria evolução das empresas que operam essas tecnologias. As interações entre essas empresas dentro do sistema estão entre os principais elementos que promovem o avanço do conhecimento tecnológico, dado que as experiências e competências de cada empresa transbordam para o conjunto do sistema (Rosenberg, 2006). De acordo com Chagas e Cabral (2010, p. 43), as capacitações desenvolvidas em integração de sistemas possuem como fundamento as capacitações funcionais (engenharia de sistemas) e as capacitações em projetos (gestão de projetos e aprendizagem organizacional).

A visão da firma baseada em recursos e das capacitações dinâmicas (Penrose, 1959; Chandler, 1990; Teece e Pisano, 1994) é complementar à análise da integração de sistemas. Para Penrose (1959, p. 176), as empresas possuem uma base produtiva e tecnológica, ou seja, um conjunto de recursos desenvolvidos internamente ao longo do tempo, complementares entre si e que se interligam no processo produtivo. A maneira como as empresas utilizam suas capacitações são essenciais para definir a posição da firma dentro da cadeia de valor de uma indústria.

No setor espacial as empresas ofertantes de sistemas complexos são as integradores finais que conectam demandantes dos sistemas espaciais — satélites e veículos lançadores — com os fornecedores de subsistemas, componentes e serviços especializados que constituem o produto final direcionado para atender a requisitos específicos dos demandantes (Guerra e Teixeira, 2001). Em resumo, no setor espacial as empresas integradoras finais comandam o conjunto da cadeia produtiva e também são as que respondem pela maior parcela de valor agregado.

\section{Os Arranjos Organizacionais do Setor Espacial Brasileiro}

Os primeiros projetos espaciais brasileiros surgiram no início da década de 1960, a partir de interesses científicos e militares específicos. Em 1979, foi instituída a Missão Espacial Completa Brasileira (MECB), buscando unificar os projetos espaciais num programa de grande porte e longo prazo. Nas décadas seguintes, o setor espacial brasileiro apresentou êxitos parciais, mas também alguns fracassos. Os projetos que mais avançaram tinham por objetivo a manufatura de satélites, com destaque para o projeto do China-Brazil Earth Resources Satellite (CBERS), que produziu cinco satélites de sensoriamento em parceria com a China, lançados entre 1999 e 2014. O setor espacial brasileiro também avançou no desenvolvimento de foguetes de sondagem, que chegaram a

\footnotetext{
${ }^{2}$ As organizações externas incluem fornecedores, usuários, órgãos governamentais, reguladores, parceiros de produção e, por vezes, concorrentes.
} 
ser exportados para outros países ${ }^{3}$. Por sua vez, os maiores fracassos estão relacionados aos Veículos Lançadores de Satélites (VLS-1), cujos três primeiros lançamentos fracassaram ${ }^{4}$. Como resultado, a capacitação desenvolvida pelo setor no Brasil é bastante heterogênea e está centrada em atividades específicas vinculadas aos principais projetos espaciais, particularmente os mais bem sucedidos.

Neste contexto, esta seção procura avaliar o arranjo organizacional do setor espacial brasileiro, evideciando suas principais características através dos dados quantitativos e informações qualitativas obtidos por meio de entrevistas realizadas junto das mais importantes empresas do setor. A pesquisa da qual se origina este texto teve como amostra onze empresas, selecionadas a partir da atuação nos principais projetos espaciais brasileiros. Essa amostra abrange empresas de variados portes e diferentes atividades, que respondem por grande parte da estrutura produtiva do setor espacial brasileiro. Durante a pesquisa, executada nos anos de 2012 e 2013, foram enviados previamente questionários e, na sequência, realizadas entrevistas in loco com os executivos que ocupavam posições chaves que pudessem fornecer as informações de suas respectivas empresas e, consequentemente, contribuissem para um maior entendimento do setor. De maneira geral, as informações coletadas demonstram como o setor está configurado, permitindo identificar as principais características de sua estrutura produtiva. De maneira geral, trata-se de um setor com competências diversificadas, de dimensões pequenas e altamente intensivo em tecnologia.

Este estudo concentrou as entrevistas nas empresas voltadas para produção das plataformas espaciais — satélites e veículos lançadores - e seus respectivos sistemas. Dado que as plataformas espaciais são equipamentos complexos que integram conjunto de sistemas altamente sofisticados, o estudo dos arranjos organizacionais está divido em duas partes. A primeira, concentrada na análise das onze empresas fornecedoras de componentes e sistemas específicos, objeto da pesquisa de campo anteriormente realizada. A segunda parte se concentra na análise da estrutura organizacional voltada para a integração das plataformas, destacando as mudanças recentes.

\subsection{Empresas fornecedoras}

As empresas da amostra vem participando como fornecedoras de componentes e sistemas dos maiores projetos espaciais brasileiros. De acordo com o Quadro 1, observa-se que há uma concentração em três grandes programas espaciais: o Satélite Sino-Brasileiro de Recursos Terrestres (CBERS), que conta com a participação de dez das onze empresas analisadas; a Plataforma Multimissão (PMM), com a participação de oito empresas; e o Veículo Lançador de Satélite (VLS), com quatro empresas. Além desses, foram indicados os programas do veículo de sondagem VBS-30 e do Humidity Sounders of Brazil (HSB), cada um contando com a participação de uma única empresa fornecedora. Por fim, cabe ressaltar que duas empresas da amostra também forneceram sistemas de solo para o Centro de Lançamento de Alcântara (CLA).

A primeira característica a ser observada é o fato da estrutura produtiva das empresas fornecedoras do setor espacial brasileiro se apresentar muito segmentada e pulverizada, refletindo a especialização de cada uma das empresas na produção de determinados sistemas, como é possível observar no quadro 1. Apenas as empresas Neuron e Ominsys participaram dos programas espaciais oferecendo o mesmo conjunto de produtos (antenas, transmissores e transponderes). Este alto grau de especialização das empresas fornecedoras decorre fundamentalmente da elevada diversidade tecnológica que envolvem os equipamentos complexos, no caso as plataformas espaciais.

\footnotetext{
${ }^{3}$ No total seis foguete de sondagem VSB-30 foram exportados para a Agência Espacial Européia (ESA), sendo lançados entre 2005 e 2013, na base de Kiruna na Suécia.

${ }^{4}$ Os dois primeiros foram destruídos no lançamento em 1997 e 1999, e o terceiro explodiu no solo acidentalmente matando 21 engenheiros que trabalhavam no programa espacial, em 2003 (Ferreira, 2010).
} 


\section{Quadro 1 - Principais empresas fornecedoras do setor espacial brasileiro: Áreas de atuação e respectivos programas}

\begin{tabular}{|c|c|c|}
\hline Empresas & Sistemas e componentes fornecidos & Programas $^{1}$ \\
\hline AEL Sistemas & Sistema de suprimento de energia elétrica & CBERS e PMM \\
\hline CENIC & Estruturas em material composto & $\begin{array}{l}\text { CBERS, PMM, VLS e VBS- } \\
\qquad 30\end{array}$ \\
\hline Compsis & Sistemas de alta tecnologia & CBERS, VLS e CLA \\
\hline Equatorial & $\begin{array}{c}\text { Câmara imageadora de amplo campo de visadade }(\mathrm{WFI})^{2} \mathrm{e} \\
\text { sensores de umidade }\end{array}$ & CBERS e HSB \\
\hline Fibraforte & Estruturas, sistemas mecânicos e de propulsão & CBERS E PMM \\
\hline Fundação Ezute & $\begin{array}{l}\text { Especificações do SGB, gerenciamento do projeto PMM e sistemas } \\
\text { de teste do VLS }\end{array}$ & SGDC, PMM e VLS \\
\hline Mectron & $\begin{array}{c}\text { Gravadores de dados digitais (DDR) e sistemas de suprimento de } \\
\text { energia, de telemetria e telecomando (TT\&C) }\end{array}$ & CBERS E PMM \\
\hline Neuron & Antenas, transmissores e transponders & CBERS e PMM \\
\hline Omnisys & Antenas, transmissores e transponders & CBERS e CLA \\
\hline Opto Eletrônica & $\begin{array}{l}\text { Câmera multiespectral de alta resolução (MUX) e de amplo campo } \\
\text { de visada (AWFI) }\end{array}$ & CBERS e PMM \\
\hline Orbital & Gerador fotovoltaico e engenharia de sistemas & CBERS, PMM e VLS \\
\hline
\end{tabular}

1. Plataforma Multimissão (PMM), Veículo Lançador de Satélite (VLS), (VBS-30), Satélite Sino-Brasileiro de Recursos Terrestres (CBERS) e Humidity Sounders of Brazil (HSB).

2. Desenvolvida em conjunto com a Opto Eletrônica.

Fonte: Elaboração própria a partir da base de dados do Mapeamento da Indústria Espacial Brasileira (CGEE).

Esta heterogeneidade também pode ser observada quando se analisa a atividade principal, segundo a CNAE das empresas fornecedoras, no Quadro 2. As onze empresas pesquisadas totalizaram oito diferentes classificações de atividade principal, a saber: desenvolvimento de programas de computador sob encomenda; fabricação de artefatos de material plástico para usos industriais; fabricação de componentes eletrônicos; fabricação de equipamento bélico pesado, exceto veículos militares de combate; fabricação de equipamentos e instrumentos ópticos, peças e acessórios; fabricação de equipamentos transmissores de comunicação, peças e acessórios; pesquisa e desenvolvimento experimental em ciências físicas e naturais e; serviços de engenharia. Observase que a maioria das empresas são intensivas em atividades de pesquisa e desenvolvimento (P\&D) e em engenharia, sendo um importante indicador de competência dessas empresas em atividades que envolvem baixa e, principalmente, média maturidade tecnológica, de acorco com o conceito de Technology Readiness Levels (TRL), desenvolvido pela National Aeronautics and Space Administration (NASA) (Mankins, 1995). 
Quadro 2 - Atividade princiapal e controle acionário das empresas pesquisadas

\begin{tabular}{|c|c|c|}
\hline Empresas & Atividade Principal (CNAE) & Controle acionário \\
\hline AEL Sistemas & Fabricação de componentes eletrônicos & $\begin{array}{l}\text { Elbit Systems Ltd }(79,84 \%) \\
\text { Embraer Defesa e Segurança }(20,16 \%)\end{array}$ \\
\hline Cenic & $\begin{array}{l}\text { Fabricação de artefatos de material plástico } \\
\text { para usos industriais }\end{array}$ & Sócios fundadores (100\%) \\
\hline Compsis & $\begin{array}{l}\text { Desenvolvimento de programas de computador } \\
\text { sob encomenda }\end{array}$ & Sócios fundadores (100\%) \\
\hline Equatorial Sistemas & $\begin{array}{l}\text { Pesquisa e desenvolvimento experimental em } \\
\text { ciências físicas e naturais }\end{array}$ & $\begin{array}{l}\text { Astrium (83\%) } \\
\text { Sócios fundadores (17\%) }\end{array}$ \\
\hline Fibraforte & Serviços de engenharia & Sócios fundadores (100\%) \\
\hline Fundação Ezute & $\begin{array}{l}\text { Pesquisa e desenvolvimento experimental em } \\
\text { ciências físicas e naturais }\end{array}$ & Fundação de direito privado nacional (100\%) \\
\hline Mectron & $\begin{array}{l}\text { Fabricação de equipamento bélico pesado, } \\
\text { exceto veículos militares de combate }\end{array}$ & $\begin{array}{l}\text { Odebrecht }(65,2 \%) \\
\text { Sócios fundadores }(34,8 \%)\end{array}$ \\
\hline Neuron & Fabricação de componentes eletrônicos & Sócios fundadores (100\%) \\
\hline Omnisys & $\begin{array}{l}\text { Fabricação de equipamentos transmissores de } \\
\text { comunicação, peças e acessórios }\end{array}$ & Grupo Thales (100\%) \\
\hline Opto Eletrônica & $\begin{array}{l}\text { Fabricação de equipamentos e instrumentos } \\
\text { ópticos, peças e acessórios }\end{array}$ & $\begin{array}{l}\text { Sócios fundadores }(83,8 \%) \\
\text { Dois fundos de investimento }(16,2 \%)\end{array}$ \\
\hline Orbital & Serviços de engenharia & Sócios fundadores (100\%) \\
\hline
\end{tabular}

Fonte: Elaboração própria a partir da base de dados do Mapeamento da Indústria Espacial Brasileira (CGEE).

Outra importante característica observada é a natureza multi-campo das atividades dessas empresas, na maioria dos casos extrapolando as fronteiras do setor espacial. A média do faturamento dessas onze empresas obtido no setor espacial foi de 37\% do total, em 2012. Entretanto observa-se uma elevada heterogeneidade nessa participação. Enquanto algumas empresas concentraram suas atividades no setor espacial — duas delas declararam ter a totalidade do seu faturamento oriunda desse setor ${ }^{5}$ — outras possuem uma participação pequena das atividades produtivas no setor espacial resultando em uma parcela menor do faturamento advinda desse setor. No entanto, como é possível observar visualmente no gráfico 1, embora as empresas possuam faturamento total muito distinto uma das outras, o faturamento relacionado ao setor espacial é relativamente próximo entre as diferentes empresas.

O mercado espacial brasileiro, constituído pela estimada participação do faturamento destas empresas em 2012, é consideravelmente pequeno. O conjunto do faturamento declarado das empresas pesquisadas foi aproximadamente R 424 milhões, sendo que cerca de $20 \%$ é constituído pelo setor espacial, totalizando R \$ 84 milhões. As empresas com maior participação nesse mercado são a Opto Eletrônica e a Omnisys que responderam respectivamente por 26\% e 20\% da somatória de receita das empresas pesquisadas. As empresas deste setor são diversas inclusive no porte. A média de funcionários das 11 empresas é de 143 funcionários, no entanto, existem desde microempresas que possuem em torno de 15 funcionários, como é o caso da Neuron e da Fibraforte,

${ }^{5}$ Cabe destacar que uma delas já havia fornecido para o setor aeronáutico. 
passando por empresas de médio porte que possuem acima de 200 funcionários, como é o caso da Opto Eletrônica, Ominsys e AEL Sistemas. Destaca-se ainda a Mectron, a única empresa de grande porte do setor com mais de 500 funcionários. Esta elevada heterogeneidade da estrutura produtiva resultou em um coeficiente de variação de $104 \%$ no porte das empresas. No entanto, a relação faturamento/funcionário demonstrou uma heterogeneidade relativamente menor, pois a média das empresas é de R\$ 254 mil por funcionário e o coeficiente de variação é de 37,3\%. Embora estas empresas se diferenciem em termos de porte (tanto em termos de faturamento, quanto no número de funcionários), há uma característica comum que as une, o desenvolvimento e fabricação de componentes e sistemas intensivos em tecnologia que demanda uma mão-de-obra altamente qualificada, composta na sua maioria por doutores, mestres e especialistas.

\section{Gráfico 1 - Faturamento das empresas do setor espacial (R\$ milhões) em 2012}

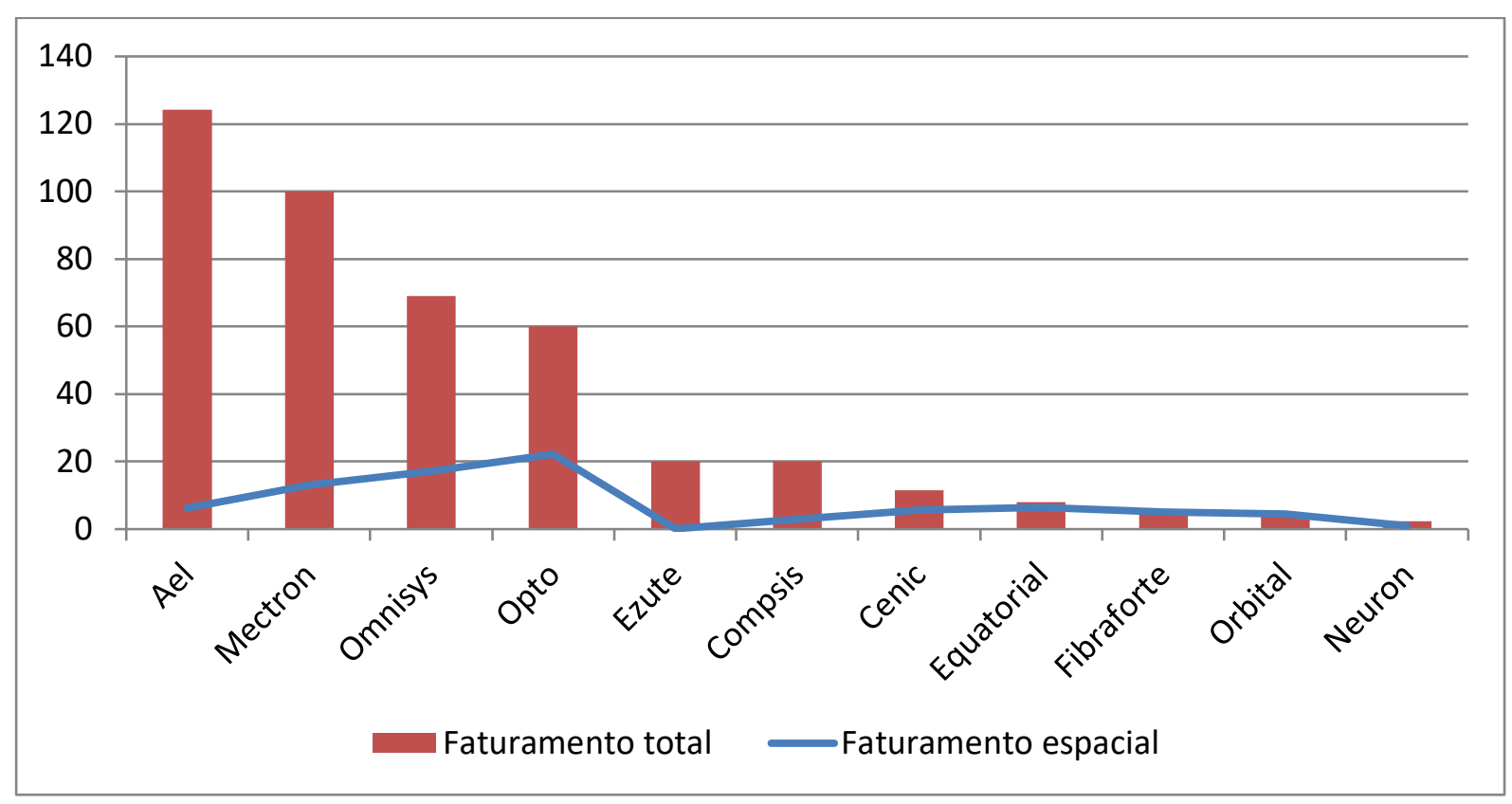

Fonte: Elaboração própria a partir da base de dados do Mapeamento da Indústria Espacial Brasileira (CGEE).

Com relação ao histórico das empresas entrevistadas, constata-se que a maioria delas iniciou as atividades no setor espacial a partir das relações com os dois princiapis centros de pesquisa em tecnologia espacial, no caso o Instituto Nacional de Pesquisas Espaciais (INPE) e o Departamento de Ciência e Tecnologia Aeroespacial (DCTA). Estas instituições, por um lado, passaram a demandar componentes e subsistemas para seus satélites e foguetes; por outro, forneciam a capacitação através do apoio dos laboratórios e pesquisadores, sendo que muitos desses pesquisadores deixaram estes centros de pesquisa para trabalharem nas empresas nacionais que estavam entrando no setor espacial, ou mesmo fundarem suas próprias empresas. Ao longo do tempo, algumas destas empresas foram sendo adquiridas por grandes grupos nacionais ou estrangeiros. Esse é o caso da empresa mais antiga da amostra, a AEL Sistemas, fundada em 1982 como Aeroeletrônica para produzir aviônicos. Na década de 1990 iniciou suas atividades no setor espacial através da demanda do INPE voltada, inicialmente, para o desenvolvimento de um sistema de suprimento de energia para o satélite de sensoriamento remoto que começava a ser construído em conjunto com a China. A capacitação da Aeroeletrônica em sistemas de energia qualificou a empresa para oferecer uma diversidade de outros sistemas e subsistemas para o setor espacial. Atualmente, a AEL Sistemas é uma joint venture controlada pela empresa israelense Elbit Systems (79,84\%) e pelo grupo Embraer (20,16\%). 
Outras empresas absolvidas por grandes grupos podem ser citadas. A brasileira Mectron é uma espécie de spin-off do Instituto Tecnológico da Aeronáutica (ITA/DCTA), uma vez que seus cinco acionistas originais se formaram nesta instituição e posteriormente foram trabalhar em diversas instituições/empresas do setor aeroespacial e de defesa, como o INPE, Avibrás, Embraer e outras. A empresa foi fundada em 1991 e no ano seguinte, 1992, foi contratada pelo INPE para desenvolver os módulos eletrônicos do terceiro Satélite de Coleta de Dados (SCD-3), dando início à sua atuação no setor espacial. Atualmente os sócios fundadores dividem entre quatro partes os $34,8 \%$ de controle que exercem sobre a empresa, sendo o restante $(65,2 \%)$ controlado pelo grupo Odebrecht. A Mectron tem uma ampla participação na área de Defesa, principalmente no segmento de mísseis. A Equatorial Sistemas, fundada em 1996, também é resultante de uma espécie de spinoff do INPE, uma vez que vários integrantes do seu quadro técnico, incluindo o presidente da empresa, vieram do referido instituto. Em 2006, a Equatorial teve 42\% do seu capital adquirido pela francesa Astrium (grupo Airbus) e, em 2011, o grupo francês passou a comandar 83\% do capital da empresa. Por fim, pode-se mencionar a Omnisys, que surgiu a partir da iniciativa de três funcionários da área técnica da Elebra, empresa nacional da área das tecnologia da informações e comunicação (TIC), que por conta da liberalização comercial brasileira enfrentou dificuldades no setor na segunda metade da década de 1990, resultando na sua falência em 1998. A Omnisys começou produzindo e integrando consoles dos sistemas de comando e controle do SISDACTA ${ }^{6}$ e na elaboração de sistemas de guerra eletrônica de diversos navios de combate da Marinha do Brasil. Esta relação estratégica com as Forças Armadas no Brasil impediram que nos anos de 2002 e 2003 o grupo francês Thales adquirisse a empresa, mas por conta das dificuldades financeiras enfrentadas pela empresa no ano de 2005, as Forças Armadas não tiveram como vetar a aquisição de $51 \%$ do capital Omnisys pelo grupo Thales. Esta participação foi crescendo ao longo do tempo até que no ano de 2012 o grupo francês assumiu a totalidade do capital da empresa brasileira, transformando-a na sua subsidiária integral.

Cabe ressaltar que, embora estas quatro empresas fossem consideradas relativamente pequenas, no momento de suas respectivas aquisições, possuíam competências essenciais que as fizeram atrativas para grupos econômicos consolidados mundialmente, indicando a significativa capacidade tecnológica que estas empresas possuíam e ainda possuem. Outra característica comum destas empresas é a diversificação para setores correlatos. Constata-se que três destas empresas AEL Sistemas, Mectron e Omnisys - concentram suas atividades nos setores aeronáutico e defesa, demonstrando que a utilização de uma base técnica análoga permite a obtenção de maiores economias de escopo (Martre, 2001). Em tese, o fato de diversificar as origens de seu faturamento, pode conferir uma vantagem em relação às outras empresas do setor, em termos de financiamento de suas atividades espaciais. Uma vez que, devido à instabilidade do orçamento espacial brasileiro, é realmente difícil manter uma estratégia de longo prazo concentrando as atividades apenas nesse setor.

Outro aspecto importante diz respeito aos grupos controladores. Observa-se que três destas empresas - AEL Sistemas, Omnisys e Equatorial Sistemas — estão inseridas nas estratégias internacionais de participação no setor espacial de suas respectivas matrizes. Por sua vez, a Mectron pertence ao grupo Odebrechet, um dos maiores e mais diversificados conglomerados empresariais brasileiros, que trabalha em setores de engenharia, infraestrutura, indústria, energia, transportes e meio ambiente. A elevada dimensão - internacional e/ou setorial — dos grupos controladores permite que estas empresas possuam fontes de capitalização em tempos de menor demanda do setor espacial, principalmente por parte da inconstância dos programas espaciais brasileiros.

Os dados coletados indicam que as empresas fornecedoras do setor espacial necessitam investir uma alta parcela do faturamento em P\&D para manterem sua competitividade. A atividade destas empresas é altamente intensiva em tecnologia, seja prestando serviços ou desenvolvendo e fabricando produtos. Quatro empresas que possuem participação do faturamento advindo do setor

\footnotetext{
${ }^{6}$ Sistema Integrado de Defesa Aérea e Controle de Tráfego Aéreo (SISDACTA).
} 
espacial acima da média (37\%) declararam uma participação P\&D/faturamento acima de 20\%. Ao menos para o período observado, 2012, é possível estabelecer uma relação de que quanto maior a participação do setor espacial nas receitas da empresa, maior a parcela de recursos destinado às atividades de $\mathrm{P} \& \mathrm{D}$, evidenciando a elevada intensidade tecnológica no campo espacial, mesmo quando comparado com os setores aeronáutico e de defesa.

\section{Tabela 1 - Faturamento, participação, número de funiconário e participação da P\&D no Faturamento, 2012, das empresas entrevistadas}

\begin{tabular}{lcccc}
\hline Empresas & Funcionários & $\begin{array}{c}\text { Faturamento } \\
\text { (R\$ milhões) }\end{array}$ & $\begin{array}{c}\text { Setor Espaciall } \\
\text { Faturamento (\%) }\end{array}$ & $\begin{array}{c}\text { P\&DI } \\
\text { Faturamento (\%) }\end{array}$ \\
\hline Ael Sistemas & 260 & 124,18 & 5,07 & 7,4 \\
Cenic & 51 & 11,5 & 50 & nd \\
Compsis & 90 & 20 & 15 & {$[5 ; 6]$} \\
Equatorial Sistemas & 35 & 8 & 80 & 30 \\
Fibraforte & 14 & 5 & 100 & 30 \\
Fundação Ezute & 120 & 20 & 0,5 & 13 \\
Mectron & 529 & 100 & 13 & nd \\
Neuron & 16 & 2,36 & 40 & 30 \\
Omnisys & 205 & 69 & 25 & 12 \\
Opto Eletrônica & 230 & 60 & 37 & 17 \\
Orbital & 25 & 4,5 & 100 & {$[15 ; 20]$} \\
\hline
\end{tabular}

Fonte: Elaboração própria a partir da base de dados do Mapeamento da Indústria Espacial Brasileira (CGEE).

Não por acaso, as empresas pesquisadas possuem um corpo técnico altamente qualificado. Isto também é corroborado pelo fato de que a divisão entre as áreas de desenvolvimento e produção destas empresas é praticamente inexistente. Nas empresas que estão atuando, predominantemente, no setor espacial, como a Fibraforte, a Orbital e a Neuron, é possível observar que, mesmo no seu reduzido número de funcionários, há uma elevada participação de mestres e doutores ${ }^{7}$. No caso das empresas com menor participação no setor espacial, como a Fundação Ezute, a AEL Sistemas e a Mectron, o nível de qualificação dos funcionários ainda é bastante significativo. Cabe destacar que uma das principais reclamações das empresas do setor com relação aos recursos humanos se deve ao fato de ter que demitir funcionários qualificados ao longo dos anos, por conta da redução das atividades em decorrência da inexistência de novos projetos. A substituição de funcionários com elevada qualificação e conhecimento acumulado nestas atividades complexas é um fator de desvantagem para estas empresas. De um lado, estes funcionários podem se realocar facilmente em outras atividades. De outro lado, a oferta de funcionários com as qualificações demandadas pelo setor espacial é muito restrita e a curva de aprendizado, em parte realizado dentro das próprias empresas, é de longo prazo.

A elevada intensidade de P\&D destas empresas é notável mesmo quando se leva em consideração a porcentagem existente nos outros setores de alta tecnologia. Em geral, as empresas do setor espacial brasileiro declararam investir uma parcela bem acima da média observada em outros setores intensivos em tecnologia. Por exemplo, De Negri e Cavalcanti (2013, p. 5) demonstraram que na indústria de transformação o setor de alta tecnologia, em média, possui uma

\footnotetext{
${ }^{7}$ Fibraforte, Orbital e Neuron possuíam, em 2012, respectivamente, 14 (nove graduados e um mestre), 25 (11 graduados, quatro mestres e um doutor) e 16 (quatro técnicos, cinco graduados e dois doutores) funcionários.
} 
relação de $1,9 \%$ dos gastos em atividades internas e externas de $P \& D$ sobre a receita líquida de vendas (PINTEC, 2011). No setor espacial, por sua vez, o menor percentual declarado por uma das empresas foi de $7,4 \%$ e o maior de $30 \%$. Cabe ainda ressaltar que neste setor os investimentos em P\&D não são a única estratégia para se capacitar tecnologicamente, dado que a maioria das empresas também declarou o aprendizado via learning by doing e a utilização dos laboratórios do INPE e do DCTA para testes, especificações e certificações básicas. Por fim, algumas empresas também declararam utilizar de engenharia reversa.

\section{Quadro 3 - Principais clientes e parceiros tecnológicos das empresas fornecedoras do setor espacial}

\begin{tabular}{|c|c|c|}
\hline Empresas & Clientes (setor espacial) & Parceiros Tecnológicos \\
\hline Ael Sistemas & AEB/INPE; Exportações & $\begin{array}{l}\text { Universidades: UFRGS, PUCRS e Unisinos } \\
\text { Centro de pesquisa: Tecnopuc }\end{array}$ \\
\hline Cenic & $\begin{array}{c}\text { AEB/INPE; DCTA; Subcontratada por Mectron e Orbital } \\
\text { Engenharia }\end{array}$ & $\begin{array}{l}\text { Universidades: ITA, USP e UFSCAR } \\
\text { Centros de pesquisa: DCTA e INPE }\end{array}$ \\
\hline Compsis & $\begin{array}{c}\text { AEB/INPE; DCTA; Subcontratada por grandes empresas } \\
\text { transnacionais }\end{array}$ & $\begin{array}{c}\text { Universidades: ITA } \\
\text { Centros de pesquisa: DCTA e INPE }\end{array}$ \\
\hline Equatorial Sistemas & AEB/INPE; DCTA & $\begin{array}{c}\text { Universidades: ITA, UNIVAP e FATEC } \\
\text { Centros de pesquisa: DCTA } \\
\text { Empresas: Airbus Military (EADS) }\end{array}$ \\
\hline Fibraforte & $\begin{array}{c}\text { AEB/INPE; Subcontratada por Orbital Engenharia, } \\
\text { Equatorial Sistemas e Cenic }\end{array}$ & $\begin{array}{c}\text { Universidades: FATEC } \\
\text { Centros de pesquisa: ITA/DCTA }\end{array}$ \\
\hline Fundação Ezute & AEB/INPE; DCTA & Offset internacional \\
\hline Mectron & AEB/INPE; DCTA & $\begin{array}{c}\text { Universidades: ITA } \\
\text { Centros de pesquisa: DCTA e INPE }\end{array}$ \\
\hline Neuron & $\begin{array}{l}\text { AEB/INPE; Subcontratada por Elebra, Omnisys e } \\
\text { Mectron }\end{array}$ & - \\
\hline Omnisys & AEB/INPE; DCTA; Exportações & Universidades: UFABC e Unicamp \\
\hline Opto Eletrônica & AEB/INPE & $\begin{array}{c}\text { Universidades: USP } \\
\text { Centros de pesquisa: CTEx/IME, IEAv/DCTA }\end{array}$ \\
\hline Orbital & $\begin{array}{c}\text { AEB /INPE; DCTA; Exportações; Subcontratação por } \\
\text { outra empresa local }\end{array}$ & $\begin{array}{c}\text { Centros de pesquisa: INPE, IAE/DCTA e } \\
\text { IEAv/DCTA }\end{array}$ \\
\hline
\end{tabular}

Fonte: Elaboração própria a partir da base de dados do Mapeamento da Indústria Espacial Brasileira (CGEE).

Devido à esta natureza altamente intensiva em tecnologia do setor, observa-se também uma intensa interação das empresas da amostra com universidades e centros de pesquisa brasileiros. ITA, USP, Unicamp, UFABC, UFSCar, UFRGS, PUC-RS, Unisinos, Univap e Fatec estão entre as instituições citadas pelas empresas. Esta interação com as universidades e centros de pesquisa é ainda mais importante na elaboração e fabricação de produtos complexos, pois as empresas não conseguem suprir sozinhas as diferentes lacunas tecnológicas encontradas no desenvolvimento e produção de componentes e subsistemas diversos. Como exemplo, é possível citar a parceria entre AEL Sistemas e a UFRGS a fim de desenvolver capacitação para a produção de componentes de tecnologia híbrida, particularmente, a UFRGS vem dando suporte à AEL em circuitos radhard (CGEE, 2013). Outro exemplo é o da empresa Opto Eletrônica. Situada na cidade de São Carlos, ela interage com a FSC-USP em física e optrônica, com a EESC-USP em aeronáutica, mecânica fina e eletrônica, com o IPEN-USP em ensaios, além do departamento de computação da UFSCar por conta dos benefícios da Lei de Informática.

Apenas três empresas declararam ter clientes internacionais, em 2012, são elas a AEL Sistemas, a Omnisys e a Orbital. Do ponto de vista do faturamento, é possível perceber que as empresas fornecedoras do setor espacial são totalmente dependentes das demandas geradas a partir 
dos projetos espaciais, como já mencionado, uma vez que todas elas declararam ter como principal cliente no setor espacial a Agência Espacial Brasileira (AEB) através do Instituto Nacional de Pesquisas Espaciais (INPE). Por se tratar de uma instituição de pesquisa o INPE também é frequentemente declarado como principal parceiro tecnológico das empresas, bem como o DCTA. Além disso, a Fundação Ezute declarou ter como parceiros tecnológicos seus offsets internacionais indicando a inserção ativa dessa uma empresa que é inteiramente brasileira.

Outra relação interessante, observada no conjunto destas empresas, é a atividade de subcontratação que está relacionada diretamente ao tipo de componente e sistema que fornecem para as integradoras das plataformas espaciais. Como forma de complementar suas atividades produtivas e de serviços, as empresas se valem da heterogeneidade que compõem a base produtiva e se valem das capacitações uma das outras.

\section{Tabela 2 - Princiapais fontes de financiamento das empresas fornecedoras do setor espacial}

\begin{tabular}{ccc}
\hline Financiamento & Empresas & Part. (\%) \\
\hline Finep & 11 & 100 \\
Fapesp & 9 & 75 \\
CNPq & 3 & 25 \\
BNDES & 3 & 25 \\
Incentivos fiscais & 6 & 50 \\
Matriz (Exterior) & 3 & 25 \\
\hline
\end{tabular}

Fonte: Elaboração própria a partir da base de dados do Mapeamento da Indústria Espacial Brasileira (CGEE).

Do ponto de vista do financiamento, é interessante notar a presença das principais agências de fomento científico e tecnológico do país como financiadoras destas projetos. Todas as empresas analisadas contam com financiamento da Financiadora de Estudos e Projetos (Finep). A Finep é a principal fonte com seus recursos de subvenção para projetos ou chamadas públicas. A Fapesp é responsável por financiar as empresas do Estado de São Paulo, principalmente através do Programa de Apoio à Pesquisa em Parceria para Inovação Tecnológica (PITE) e o CNPq, através de chamadas públicas, financiou três empresas da amostra. Seis empresas declararam ter como financiamento os incentivos fiscais, como a Lei de Informática, a Lei do Bem e a Lei da Inovação, valendo-se do fato de que também atuam em outros setores que não só o espacial. As três empresas que possuem capital internacional também contam com financiamento advindo da matriz. O Banco Nacional de Desenvolvimento Econômico e Social (BNDES) financia os investimentos das grandes empresas com ou através da entrada do BNDESPar dando suporte aos programas de consolidação dessas empresas, sendo este o caso da aquisição da Mectron pelo grupo Odebrecht. A atuação do BNDES junto das três maiores empresas fornecedoras insere mais uma vez um elemento de heterogeneidade que caracteriza as empresas do setor.

\subsection{As instituições integradoras e a criação da Visiona}

No caso brasileiro, desde a década de 1970, a integração das plataformas espaciais é realizada pelo Estado, através dos dois principais centros de pesquisa do setor. O Instituto Nacional de Pesquisas Espaciais (INPE), criado em 1971, e atualmente vinculado ao Ministério da Ciência, Tecnologia, Inovações e Comunicações (MCTIC), é a instituição responsável pelo projeto e integração dos satélites. Além disso, atividades operacionais e de manutenção em relação à infraestrutura, desenvolvimento, integração, testes, rastreamento e controle de satélites, recepção, processamento e distribuição de dados de satélite são de responsabilidades do instituto (Dewes e 
Padula, 2012) ${ }^{8}$. Por sua vez, o Departamento de Ciência e Tecnologia Aeroespacial (DCTA), órgão do Comando da Aeronáutica do Ministério da Defesa (MD), é responsável pelo projeto e integração dos veículos lançadores e de sondagem, através do Instituto de Aeronáutica e Espaço (IAE). Cabe destacar que o DCTA também é responsável pelo controle dos centros de lançamento de foguetes.

As atividades destes dois institutos - INPE e DCTA — vem delimitando as relações entre os atores do setor espacial e o arranjo organizacional do qual fazem parte. Estes institutos são os únicos contratantes da indústria espacial brasileira e, além disso, também atuam como os principais parceiros tecnológico das empresas do setor, como é possível notar no quadro 3.

Contudo, uma importante mudança neste arranjo organizacional teve início em 2012 com a criação da Visiona Tecnologia Espacial SA. Essa empresa foi concebida a partir de uma iniciativa do governo federal de estabelecer uma empresa integradora nacional na indústria espacial que atendesse particularmente as demandas públicas, tanto civis como militares, expressas, respectivamente, no Programa Nacional de Atividades Espaciais (PNAE) e na Estratégia Nacional de Defesa (END).

Neste contexto, a Visiona foi constituída como uma joint venture entre a Embraer, grupo brasileiro líder nos setores aeroespacial e de defesa, que detém $51 \%$ do capital da nova empresa, e a Telebras, empresa estatal de economia mista do setor de telecomunicações, com $49 \%$ do capital (Visiona, 2016). A empresa está atuando como a principal contratante (prime contractor) do programa do Satélite Geoestacionário de Defesa e Comunicações Estratégicas (SGDC), que visa garantir a segurança das redes de telecomunicações do governo federal, além de permitir a ampliação do Programa Nacional de Banda Larga (PNBL) para as regiões mais isoladas do país, como a Amazônia e o interior do Nordeste.

Em 2013 a Visiona assinou um contrato de R\$ 1,3 bilhão que prevê a entrega do primeiro satélite, o SGDC-1, até fevereiro de 2017. Como principal contratante, a Visiona realizou uma seleção internacional e contratou a empresa franco-italiana Thales Alenia Space e a europeia Arianespace, para fornecerem, respectivamente o satélite e seu lançamento à órbita. No contrato com a Thales Alenia Space (TAS) também está previsto a transferência de tecnologia para empresas brasileiras, particularmente no que se refere a integração de sistemas (Infodefesa, 2016).

Para atender esta demanda, a Visiona está sendo formada com profissionais egressos da Embraer e do INPE, acrescido de jovens engenheiros que estão diretamente envolvidos nas atividades de projeto e produção do SGDC (on job training) através de um Programa de Absorção de Tecnologia nas unidades da TAS localizadas na França (Visiona, 2016). As competências buscadas por este programa estão concentradas principalmente em engenharia de sistemas, gerenciamento de projetos, gestão de riscos, especificação e gestão de requisitos, cujo objetivo final é garantir a consolidação da Visiona como a empresa integradora da indústria espacial nacional.

Dentro do contrato de aquisição do SGDC junto da TAS também foi estabelecido um Plano de Transferência de Tecnologia coordenado pela AEB com o objetivo de beneficiar as empresas nacionais estabelecidas no setor espacial. Contudo, constata-se que a participação dos fornecedores nacionais na construção do SGDC-1 é considerada insignificante. Há um consenso de que a urgência que o programa SGDC passou a ter praticamente impediu a participação das empresas brasileiras. Além disso é importante destacar que um satélite geoestacionário de comunicações é um projeto com grandes diferenças dos projetos de satélites de sensoriamento remoto e coleta de dados, únicos modelos produzidos no Brasil.

\footnotetext{
${ }^{8}$ Em 2015, visando impulsionar projetos de desenvolvimento de novas tecnologias, sistemas e equipamentos, o INPE selou um acordo junto com a Fapesp para selecionar empresas que mandassem propostas dentro do Programa Fapesp Pesquisa Inovativa em Pequenas Empresas (PIPE). Observa-se que algumas empresas da amostra se beneficiaram desse acordo e tiveram nele uma importante fonte de financiamento.
} 


\section{Breves Considerações}

Na maioria das empresas fornecedoras analisadas, a participação no setor espacial é, em geral, uma atividade minoritária, particularmente das maiores. Apesar dessa atuação restrita, a maioria das empresas fornecedoras apresenta uma destacada capacitação em tecnologias de sistemas e componentes especializados, cujo volume de produção é pequeno. Desta maneira, estas empresas apresentam baixas economias de escalas, mas razoáveis economias de escopo, pois utilizam a mesma base tecnológica para desenvolver produtos em setores correlatos. Neste sentido, o presente trabalho procurou demonstar as principais características das empresas fornecedoras, pontuando alguns problemas decorrentes da heterogeneidade que essas empresas possuem em termos de financiamento e faturamento e como o papel do Estado é fundamental para capacitação dessas empresas.

Com relação às mudanças nos arranjos organizacionais, destaca-se o papel da Visiona como uma nova integradora e contratante de um novo programa espacial, o SGDC. Por se tratar de um trabalho ainda em andamento, as próximas etapas desta pesquisa consistem em observar como esta empresa vem atuando como uma nova integradora do setor, como vem se posicionando e alterando o arranjo organizacional da indústria espacial brasileira. De que maneira a Visiona atuará, se está contribuindo para o fortalecimento e adensamento do setor espacial ou se sua atuação vem corroborando para debilitar, ou mesmo extinguir, empresas fornecedoras já existentes.

\section{Referências Bibliográficas}

AGÊNCIA ESPACIAL BRASILEIRA - AEB (2016). Informações Institucionais. Disponível em: http://www.aeb.gov.br/. Acessado em abril de 2016.

CARVALHO, H. (2011). Alternativas de Financiamento e Parcerias Internacionais Estratégicas no Setor Espacial. In: BRASIL. Presidência da República. Secretaria de Assuntos Estratégicos. Desafios do Programa Espacial Brasileiro. Brasília: SAE, 2011.

CHAGAS, M.; CABRAL, A. (2010) Criação de capacitações em integração de sistemas: o caso do programa CBERS. Revista de Administração e Inovação, v. 7, n. 2, pp. 34-59, abr./jun.

CHAGAS, M.; CABRAL, A.; CAMPANÁRIO, M. (2011) Firmas Integradoras de Sistemas, suas Capacitações e Fontes de Tecnologia - O Caso da Empresa Brasileira de Aeronáutica (Embraer). Revista de Ciências da Administração, v. 13, n. 29, p. 63-87.

CHANDLER, A. (1990) Scale and scope: the dynamics of industrial capitalism. Cambridge: Harvard University Press.

DE NEGRI, F.; CAVALCANTE, L. (2011) Análise de dados da Pintec 2011. Nota técnica, n. 15. Brasília: Ipea.

DEWES, M. (2012) Projetos nacionais de inovação: práticas do setor espacial brasileiro. Tese (Doutorado em Administração), Universidade Federal do Rio Grande do Sul, Porto Alegre.

DEWES, M.; PADULA, A. (2012) Innovation in a strategic development program: the Aeroespace Program in Brazil. Revista Brasileira de Inovação, v. 11, n. 1, p. 169-194, jan-jun.

FERREIRA, M.J.B. (2009) Dinâmica da inovação e mudanças estruturais: um estudo de caso da indústria aeronáutica mundial e a inserção brasileira (Tese de Doutorado). Campinas: Instituto de Economia, Universidade Estadual de Campinas (IE/Unicamp).

FERREIRA, M.J.B. (2010) A industrial espacial brasileira: inserção, desafios e oportunidade. Boletim Neit, n. 15, jan-abr, pp. 1-9.

FUTRON (2012). Futron's 2012 Space Competitiveness Index: a comparative analysis off how countries invest in and benefit from space industry. Futron Corporation.

GATTAZ, C.; CATHARINO, M.; VAZ, C. (2012) O caso do sistema setorial de inovação e produção espacial brasileiro: desafios para a gestão de uma rede de cooperação. In: OLIVEIRA, 
V.F.; CAVENAGHI, V.; MÁSCULO, F.S. (Orgs.). Tópicos emergentes e desafios metodológicos em Engenharia de Produção: casos, experiências e proposições. V.V, ABEPRO.

GUERRA, O.; TEIXEIRA, F. (2011) Da oferta de Sistemas Complexos de Produção para o Suprimento de Serviços e Sistemas Integrados: uma Trajetória Evolutiva. Anais do XXIX Encontro da Associação Nacional dos Centros de Pós-Graduação em Economia (ANPEC), Salvador (BA), 11-14 de dezembro.

HOBDAY, M.; DAVIES, A.; PRENCIPE, A. (2005) Systems integration: a core capability of the modern corporation. Industrial and Corporate Change, v. 14, n. 6, p. 1109-1143.

INFODEFESA (2016) Informações institucionais. Disponível em: http://www.infodefensa.com/latam/2013/08/28/noticia-visiona-seleciona-empresas-fornecedorasdo-sistema-do-satelite-geoestacionario-brasileiro-sgdc.html. Acessado em abril de 2016.

MATOS, P. (2016). Sistemas espaciais voltados para Defesa. In Mapeamento da Base Industrial de Defesa. Brasília: ABDI - Agência Brasileira de Desenvolvimento Industrial: Ipea - Instituto de Pesquisa Econômica Aplicada, pp. 509-595.

MANKINS, J.C. (1995). Technology Readiness Levels: A White Paper. NASA, Office of Space Access and Technology, Advanced Concepts Office.

MARTRE, H. (2001). A indústria aeroespacial: Análises e reflexões. Disponível em: http://www.france.org.br/abr/imagesdelafrance/aeroespacial.htm. Acessado em: 2016.

MILLER, R.; HOBDAY, M.; LEROUX-DEMERS, T.; OLLEROS, X. (1995) Innovation in Complex Product Systems Industries: the case of flight simulation. Industrial and Corporate Change, v. 4, n. 2, p. 363-400, 1995.

PENROSE, E. (2006 [1959]) A teoria do crescimento da firma. Campinas: Editora da Unicamp.

ROSENBERG, N. (2006 [1982]) Por dentro da caixa preta. Campinas: Editora da Unicamp.

TEECE, D.; PISANO, G. (1994) The dynamic capabilities of firms: an introduction. Industrial and Corporate Change, v. 3, n. 3, p. 537-556.

UTTERBACK, J. (1996) Dominando a Dinâmica da Inovação. Rio de Janeiro: Qualitymark Editora.

VAZ, C. (2011) Fomento e apoio ao desenvolvimento da capacidade industrial, atendimento às demandas de fabricação dos projetos espaciais. In: BRASIL. Presidência da República. Secretaria de Assuntos Estratégicos. Desafios do Programa Espacial Brasileiro. Brasília: SAE.

VISIONA TECNOLOGIA ESPACIAL (2016) Informações institucionais. Disponível em: http://www.visionaespacial.com.br/. Acessado em abril de 2016. 